EPJ Web of Conferences 81, 05021 (2014)

DOI: 10.1051/epjconf/ 20148105021

(C) Owned by the authors, published by EDP Sciences, 2014

\title{
Description of hadrons with Covariant Quark Model
}

\author{
A. Liptaj ${ }^{1, a}$, M. A. Ivanov², S. Dubnička ${ }^{1}$, and A. Z. Dubničková3 \\ ${ }^{1}$ Institute of Physics, Slovak Academy of Sciences, Bratislava, Slovakia \\ ${ }^{2}$ Bogoliubov Laboratory of Theoretical Physics, Joint Institute for Nuclear Research, Dubna, Russia \\ ${ }^{3}$ Faculty of Mathematics, Physics and Informatics, Comenius University, Bratislava, Slovakia
}

\begin{abstract}
Covariant quark model represents an effective field approach to hadronic interactions. It is based on a non-local Lagrangian, which provides full Lorentz invariance and has limited number of parameters. The model is suitable for multi-quark state description and successfully describes numerous experimental measurements. In this text we give a brief introduction to the model and refer to the achieved results.
\end{abstract}

\section{Introduction}

Hadron physics, nowadays in center of interest thanks to heavy-quark factories, lacks solid firstprinciple theoretical explanation for the wide range of the measured data. The perturbative approach is no longer applicable and other approaches with small model dependence (lattice QCD, ChPT) are usually restrained to a specific phase-space region.

To cover the hadronic experimental data in their large variety a model-depended approach is needed. The covariant quark model (CQM) can be applied to wide spectra of hadronic processes and results can be calculated using standard quantum-field theory techniques.

\section{Covariant quark model in a nutshell}

The CQM introduces an effective quark-hadron interaction (no gluons) which is governed by following Lagrangian (density)

$$
\mathcal{L}_{\mathrm{int}}=g_{H} \cdot H(x) \cdot J_{H}(x),
$$

where the current $J_{H}(x)$ for different hadrons (meson, baryon, tetraquark) is

$$
\begin{aligned}
J_{M}(x)= & \int d x_{1} \int d x_{2} F_{M}\left(x, x_{1}, x_{2}\right) \cdot \bar{q}_{f_{1}}^{a}\left(x_{1}\right) \Gamma_{M} q_{f_{2}}^{a}\left(x_{2}\right), \\
J_{B}(x)= & \int d x_{1} \int d x_{2} \int d x_{3} F_{B}\left(x, x_{1}, x_{2}, x_{3}\right) \times \Gamma_{1} q_{f_{1}}^{a_{1}}\left(x_{1}\right)\left(q_{f_{2}}^{a_{2}}\left(x_{2}\right) C \Gamma_{2} q_{f_{3}}^{a_{3}}\left(x_{3}\right)\right) \cdot \varepsilon^{a_{1} a_{2} a_{3}}, \\
J_{T}(x)= & \int d x_{1} \ldots \int d x_{4} F_{T}\left(x, x_{1}, \ldots, x_{4}\right) \\
& \times\left(q_{f_{1}}^{a_{1}}\left(x_{1}\right) C \Gamma_{1} q_{f_{2}}^{a_{2}}\left(x_{2}\right)\right) \cdot\left(\bar{q}_{f_{3}}^{a_{3}}\left(x_{3}\right) \Gamma_{2} C \bar{q}_{f_{4}}^{a_{4}}\left(x_{4}\right)\right) \cdot \varepsilon^{a_{1} a_{2} c} \varepsilon^{a_{3} a_{4} c} .
\end{aligned}
$$

\footnotetext{
ae-mail: Andrej.Liptaj@savba.sk
} 
Here $C$ is charge conjugation matrix and $F$ is a non-local vertex function

$$
F_{H}\left(x, x_{1}, \ldots, x_{n}\right)=\delta\left(x-\sum_{i=1}^{n} w_{i} x_{i}\right) \Phi_{H}\left(\sum_{i<j}\left(\left(x_{i}-x_{j}\right)^{2}\right),\right.
$$

where we require the barycenter of the quark system to correspond to the hadron position $w_{i}=m_{i} / \sum_{j=1}^{n} m_{j}$ and assume a Gaussian form of the interaction strength $\bar{\Phi}_{H}\left(-k^{2}\right)=\exp \left(k^{2} / \Lambda_{H}^{2}\right)$ in the momentum space. $\Lambda_{H}$ is a free hadron-size related parameter of the model. In addition the model contains 5 extra parameters (four quark masses and one universal cutoff), so, to describe physics with $N$ hadrons, $N+5$ parameters are required. The parameters were tuned to basic observables (leptonic decay constants, EM decay widths) giving the parameter values (in $\mathrm{GeV}$ ): $m_{u, d}=0.235, m_{s}=0.424, m_{c}=2.16, m_{b}=5.09, \lambda_{\text {cut-off }}=0.181, \Lambda_{\pi}=0.87$, etc.

We use so-called compositeness condition to achieve an appropriate description of hadrons as quark bound states and eliminate quark-hadron couplings as free parameters. This topic was studied already decades ago $[1,2]$ and the out-coming condition can be formulated as follows: the renormalization constant $Z_{M}^{1 / 2}$ can be interpreted as the matrix element between a physical state and the corresponding bare state. Requiring $Z_{M}^{1 / 2}=\left\langle M_{\text {bare }} \mid M_{\text {dressed }}\right\rangle=0$ then implies that the physical state does not contain bare state and is therefore properly described as bound. The condition can be expressed via the derivative of the hadron mass operator, which can be calculated within the CQM

$$
Z_{H}=1-\frac{3 g_{H}^{2}}{4 \pi^{2}} \tilde{\Pi}_{H}^{\prime}\left(m_{H}^{2}\right)=0
$$

The already mentioned cut-off parameter $\lambda$ was introduced into the model in order to provide an infrared cut-off and ensure stability of heavy hadrons. Unity in form of delta function $1=\int_{0}^{\infty} d t \delta\left(t-\sum_{i=1}^{n} \alpha_{i}\right)$ is introduced when evaluating Feynman diagrams, where $\alpha_{i}$ corresponds to Schwinger parameters (used to express quark propagators). Then a multidimensional improper integral can be transformed into a form where only one improper integration remains. The cut-off is afterwards applied on the upper integration limit

$$
\Pi=\int_{0}^{\infty} d^{n} \alpha F\left(\alpha_{1}, \cdots, \alpha_{n}\right)=\int_{0}^{\infty \rightarrow \frac{1}{\lambda^{2}}} d t t^{n-1} \int_{0}^{1} d^{n} \alpha \delta\left(1-\sum_{i=1}^{n} \alpha_{i}\right) F\left(t \alpha_{1}, \ldots, t \alpha_{n}\right) .
$$

In this way $\Pi$ becomes a smooth function where thresholds in the quark loop diagrams and corresponding branch points are removed. The integration is done numerically.

The objects (mostly form factors) needed to predict observable properties of hadrons come from evaluation of corresponding Feynman graphs and few smart operator identities can be used to make the evaluation effective. They can be found, e.g., in [3].

When a weak hadron decay is concerned, we use an effective theory with Wilson coefficients and four-quark vertex to describe the flavour transition.

\section{Overview of results}

The following non-exhaustive overview of chosen results demonstrates the wide application range of the CQM. 
MESON 2014 $-13^{\text {th }}$ International Workshop on Production, Properties and Interaction of Mesons

Table 1. Branching ratios (\%) for $B_{s}$ decays to charmed mesons.

\begin{tabular}{lll}
\hline Process & CQM & Experiment [8] \\
\hline$B_{s} \rightarrow D_{s}^{-} D_{s}^{+}$ & 1.65 & $1.04_{-0.26}^{+0.29}$ \\
$B_{s} \rightarrow D_{s}^{-} D_{s}^{*+}+D_{s}^{*-} D_{s}^{+}$ & 2.40 & $2.8 \pm 1.0$ \\
$B_{s} \rightarrow D_{s}^{*-} D_{s}^{*+}$ & 3.18 & $3.1 \pm 1.4$ \\
$B_{s} \rightarrow J / \Psi \Phi$ & 0.16 & $0.15 \pm 0.05$ \\
\hline
\end{tabular}

Table 2. Magnetic moment and (squared) electric and magnetic radius for proton and neutron.

\begin{tabular}{lll}
\hline Quantity & CQM & Experiment [8] \\
\hline$\mu_{p}$ (in n.m.) & 2.96 & 2.793 \\
$\mu_{n}$ (in n.m.) & -1.83 & -1.913 \\
$r_{E}^{p}(\mathrm{fm})$ & 0.805 & $0.8768 \pm 0.0069$ \\
$<r_{E}^{2}>^{n}\left(\mathrm{fm}^{2}\right)$ & -0.121 & $-0.1161 \pm 0.0022$ \\
$r_{M}^{p}(\mathrm{fm})$ & 0.688 & $0.777 \pm 0.013 \pm 0.010$ \\
$r_{M}^{n}(\mathrm{fm})$ & 0.685 & $0.862_{-0.008}^{+0.009}$ \\
\hline
\end{tabular}

\subsection{Mesons}

To illustrate results of the model in the domain of mesons one can mention the non-leptonic $B_{s}$ decays. In paper [4] we studied the processes $B_{s} \rightarrow J / \psi+\eta^{\left({ }^{\prime}\right)}$. We obtained values for branching fractions in good agreement with experimental ones $[5,6]$

$$
\begin{array}{ll}
\mathcal{B}_{\mathrm{CQM}}(\mathrm{J} / \psi \eta)=4.67 & \mathcal{B}_{\text {Belle }}(\mathrm{J} / \psi \eta)=5.10 \pm 1.12 \\
\mathcal{B}_{\mathrm{CQM}}\left(\mathrm{J} / \psi \eta^{\prime}\right)=4.04 & \mathcal{B}_{\text {Belle }}\left(\mathrm{J} / \psi \eta^{\prime}\right)=3.71 \pm 0.95
\end{array}
$$

and we have shown the importance of the non-trivial contribution of the model calculations to the corresponding form factors

$$
\begin{aligned}
& R=\frac{\Gamma\left(J / \psi+\eta^{\prime}\right)}{\Gamma(J / \psi+\eta)}=\left\{\begin{array}{ll}
0.73 \pm 0.14 \pm 0.02 & \text { Belle } \\
0.90 \pm 0.09_{-0.02}^{+0.06} & \text { LHCb }
\end{array},\right. \\
& R^{\text {theor }}=\underbrace{\frac{\left|\mathbf{q}_{\eta^{\prime}}\right|^{3}}{\left|\mathbf{q}_{\eta}\right|^{3}} \tan ^{2} \delta \times \underbrace{\left(\frac{F_{+}^{B_{s} \eta^{\prime}}}{F_{+}^{B_{s} \eta}}\right)^{2}}_{\approx 0.83} \approx 0.86 .}_{\approx 1.04}
\end{aligned}
$$

Branching fractions for different non-leptonic $B_{s}$ decays were studied in [7]. Although the results (Tab. 1) are mostly outside the $1 \sigma$ measurement uncertainties, they describe the data behavior fairly well.

\subsection{Baryons}

The CQM is suitable to study also light hadros, as demonstrated in [9]. Here, basic nucleon properties were evaluated and compared to experimental values [8], see Tab. 2.

Rare decays of heavy baryon $\Lambda_{b}$ were studied in [10]. The model prediction for the branching fraction of the semileptonic decay $\mathcal{B}\left(\Lambda_{\mathrm{b}} \rightarrow \Lambda \mu^{+} \mu_{-}\right)=1.0 \times 10^{-6}$ is close to the experimental values published by $\operatorname{CDF}(1.73 \pm 0.69) \times 10^{-6}[11]$ and $\operatorname{LHCb}(0.96 \pm 0.25) \times 10^{-6}[12]$. 


\subsection{Tetraquarks}

The covariant quark model was applied also to the case of tetraquark. Several arguments were raised to support the tetraquark character of the $X(3872)$ state. Assuming the diquark hypothesis $\left(D^{0} D^{* 0}\right.$ molecule), chosen branching fractions of this state were studied in the framework of the CQM [13, 14]. The CQM roughly describes the values measured by experiments:

$$
\frac{\Gamma(X \rightarrow \gamma J / \psi)}{\Gamma(X \rightarrow J / \psi 2 \pi)}=\left\{\begin{array}{ll}
0.15 \pm 0.03 & \mathrm{CQM} \\
0.14 \pm 0.05 & {[15]} \\
0.22 \pm 0.06 & {[16]}
\end{array} \quad \frac{\Gamma\left(X \rightarrow D^{0} \bar{D}^{0} \pi^{0}\right)}{\Gamma\left(X \rightarrow J / \psi \pi^{+} \pi^{-}\right)}= \begin{cases}4.5 \pm 0.2 & \mathrm{CQM} \\
10.5 \pm 4.7 & {[17]}\end{cases}\right.
$$

\section{Summary and conclusion}

Covariant quark model is a successful model with wide application spectra. It can be used for heavy and light hadrons, for strong and weak decays and for hadrons with different quark multiplicities (meson, baryon and tetraquark).

\section{Acknowledgements}

The work was partly supported by Slovak Grant Agency for Sciences VEGA, grant No. 1/0158/13 (S. Dubnička, A. Z. Dubničková, A. Liptaj), by Slovak Research and Development Agency APVV, grant No. APVV-046312 (S. Dubnička, A. Z. Dubničková, A. Liptaj) and by Joint research project of Institute of Physics, SAS and Bogoliubov Laboratory of Theoretical Physics, JINR, No. 01-3-1070 (S. Dubnička, A. Z. Dubničková, M. A. Ivanov and A. Liptaj).

\section{References}

[1] A. Salam, Nuovo Cim. 25 (1962) 224

[2] S. Weinberg, Phys. Rev. 130 (1963) 776

[3] M. A. Ivanov, PoS Baldin -ISHEPP-XXI (2012) 057 [arXiv:1301.4849 [hep-ph]]

[4] S. Dubnicka et al. Phys. Rev. D 87 (2013) 074201

[5] J. Li et al. [Belle Collaboration], Phys. Rev. Lett. 108 (2012) 181808

[6] R. Aaij et al. [LHCb Collaboration], Nucl. Phys. B 867 (2013) 547

[7] M. A. Ivanov et al. Phys. Rev. D 85 (2012) 034004

[8] K. Nakamura et al. [Particle Data Group Collaboration], J. Phys. G 37 (2010) 075021

[9] T. Gutsche et al. Phys. Rev. D 86 (2012) 074013

[10] T. Gutsche et al. Phys. Rev. D 87 (2013) 074031

[11] T. Aaltonen et al. [CDF Collaboration], Phys. Rev. Lett. 107 (2011) 201802

[12] R. Aaij et al. [LHCb Collaboration], Phys. Lett. B 725 (2013) 25

[13] S. Dubnicka et al. Phys. Rev. D 81 (2010) 114007

[14] S. Dubnicka et al. Phys. Rev. D 84 (2011) 014006

[15] K. Abe et al. [Belle Collaboration], hep-ex/0505037

[16] E. Klempt and A. Zaitsev, Phys. Rept. 454 (2007) 1

[17] C. Amsler et al. [Particle Data Group Collaboration], Phys. Lett. B 667 (2008) 1 